\section{CARBOHYDRATE METABOLISM}

\section{Measuring gluconeogenesis (GNG) with $\left[2-{ }^{13} \mathrm{C}\right]$ glycerol and mass isotopomer distribution analysis (MIDA) in normal rats and in rats with streptozocin- induced diabetes. O Peroni, M Odeon, $\checkmark$ Large, M Beylot (Faculté de médecine, René-Laënnec, Inserm unité 197, rue Guil- laume-Paradin, 69008 Lyon, France)}

MIDA is a new method for measuring, with stable isotope labelled precursors, the biosynthesis of compounds synthesized from the addition of several identical or comparable precursor molecules, such as glucose from two triosephosphates. It does this by allowing the calculation of the enrichment of the precursor pool.

In vitro, this method is valid. We have used it in vivo to quantify the total GNG in control rats $(n=6)$ and in rats with streptozocin-induced diabetes $(n=6)$. All rats were studied in the postabsorptive state. We used $\left[3-{ }^{3} \mathrm{H}\right]$ glucose for measuring the hepatic glucose production (HGP) and $\left[2-{ }^{13} \mathrm{C}\right]$ glycerol as a gluconeogenic tracer. Using GC$\mathrm{MS}$, the total ${ }^{13} \mathrm{C}$ glucose enrichment (IE) and glucose isotopomer distribution were measured to calculate the contribution of GNG to HGP.

In diabetic rats, glycaemia and HGP levels were higher than in control $(26.4 \pm 0.8$ vs $8.6 \pm 0.2 \mathrm{mM}, P<0.05$ and $109 \pm 6$ vs $67 \pm$ $3 \mu \mathrm{mol} / \mathrm{kg} / \mathrm{min}, P<0.05)$. The contribution of GNG to HGP was increased ( $67 \pm 3$ vs $53 \pm$ $4 \%, P<0.05)$ and the absolute GNG level was twice as high $(73 \pm 5$ vs $37 \pm 4$ $\mu \mathrm{mol} / \mathrm{kg} / \mathrm{min}, P<0.05$ ) while nongluconeogenic glucose production remained unchanged ( $36 \pm 3$ vs $31 \pm 2 \mu \mathrm{mol} / \mathrm{kg} / \mathrm{min}, P$ $<0.05$ ).

We conclude that i) the results obtained in control rats support, contrary to the recent report of Previs et al [J Biol Chem (1995) $270,19806]$, the validity of this method for in vivo studies, and ii) in this experimental model of diabetes, the increase in HGP was due to an increase in total GNG.

Role of unsaturated fatty acids in the in vitro inhibition of glucose- 6 phosphatase by glycogen. $N$ Daniele 1 , JC Bordet 2 , G Mithieux ${ }^{1}$ ( 1 Inserm U 449; 2 331, Faculté de médecine René-Laënnec, 69372 Lyon cedex 08, France)

It has been suggested that glycogen, or an endogenous-associated compound, of molecular mass $<5 \mathrm{kDa}$ and that can be dissociated by ion-exchange, could inhibit glucose-6 phosphatase (Glc6Pase) and could play a regulatory role in hepatic glucose production during nutritional transitions.

Glycogen has been purified from the liver of fed rats by $\mathrm{KOH}$ digestion and ethanol precipitation. It inhibited Glc6Pase activity in the microsomes isolated from $48 \mathrm{~h}$-fasted rats $(66 \pm 14 \%$ of control activity in the presence of $30 \mathrm{mM}$ glucosyl, mean $\pm \mathrm{SEM}, n=$ 4). After filtration on DOWEX 1 (200-400 mesh), glycogen lost its inhibitory effect on Glc6Pase (101 $\pm 3 \%$ of control). The fractions bound to the column were eluted by $30 \mathrm{mM}$ and $1 \mathrm{M} \mathrm{HCl}$, successively. After evaporation to dryness and resuspension, these fractions inhibited Glc6Pase activity ( $80 \pm 2$ and $76 \pm 3 \%$ of control, respectively).

Since glycogen is associated with the liver membranes, a hypothesis suggesting that the inhibitory compound could be of a lipidic nature was tested. Lipids were extracted from glycogen by Folch's method. Analysis by thin-layer chromatography revealed the presence of significant amounts of fatty acids (FA), but no complex lipids. Quantification by gas chromatography revealed that glycogen contained $4.9 \pm 0.7$ nmol FA/ $\mu$ mol glucosyl. After DOWEX filtration, $1.3 \pm 0.7 \mathrm{nmol}$ remained tightly bound to the glycogen and $1.2 \pm 0.6$ and 
$1.1 \pm 0.2$ were bound to the column and were eluted by $30 \mathrm{mM}$ and $1 \mathrm{M} \mathrm{HCl}$, respectively.

We concluded that the amount of unsaturated FA in each fraction $(\approx 50 \%$ of FA bound to glycogen, $\approx 90 \%$ of $F A$ in eluted fractions) may have accounted for the inhibition of Glc6Pase since it was shown that free but not bound unsaturated FA could inhibit the enzyme in vitro in relevant concentrations.

\section{Effects of lipid infusion on postabsorp-} tive glycemia in non insulin dependent diabetes mellitus (NIDDM). $V$ Rigalleau, E De Tinguy, A Iron, J Aubertin, H Gin (Clinique médicale, Tripode, 33000 Bordeaux, France)

As proposed by Randle, lipid-glucid interactions may play a role in the hyperglycemia of NIDDM. We previously reported a hyperglycemic effect of a lipid infusion in the postabsorptive state in NIDD patients [Rigalleau (1994) Metabolism 43, 1300]. This paper describes how we studied its mechanism in 30 NIDD patients. Fifteen received a 180 min lipid infusion ('Ivélip 20\%'; 0.015 $\mathrm{mL} / \mathrm{kg} / \mathrm{min}$ ) and 15 received saline (controls). Glucose, TGs, FFAs levels were measured and continuous indirect calorimetry was performed beginning $30 \mathrm{~min}$ before the start of the infusion and continuing to the end of the testing period. Lipid infusion significantly $(P<0.05)$ slowed the postabsorptive glycemic decline (lipid: $10.5 \pm 1.1$ $\mathrm{mmol} / \mathrm{L}$ to $10.2 \pm 1.0$ at $180 \mathrm{~min}$, NS; saline: $11.2 \pm 0.8$ to $9.8 \pm 0.7, P<0.01$ ). The response was heterogeneous, six 'responders' showed an absolute hyperglycemic response $(10.8 \pm 1.0$ to $11.8 \pm 0.8)$, while nine 'nonresponders' did not, in a similar manner to the saline controls $(11.4 \pm 1.2$ to $10.5 \pm 1.1$ ). The lipidic effects of the lipid infusion differed in these two groups. In 'responders', the lipid infusion produced a greater $(P<0.01)$ increase in FFAs ('responders': $839 \pm 218 \mu \mathrm{mol} / \mathrm{L}$ to $3335 \pm 840$; 'nonresponders': $954 \pm 109$ to $1731 \pm 206$ ). In contrast, TGs ('responders': $2.20 \pm$ $0.19 \mathrm{mmol} / \mathrm{L}$ to $8.56 \pm 1.54$; 'nonresponders': $1.76 \pm 0.18$ to $6.67 \pm 0.89$; NS), total lipid oxidation ('responders': $0.74 \pm$ $0.09 \mathrm{mg} / \mathrm{kg} / \mathrm{min}$ to $0.90 \pm 0.06$; 'nonresponders': $0.59 \pm 0.16$ to $0.79 \pm 0.10$; NS) and glucose oxidation ('responders': $1.27 \pm$ $0.28 \mathrm{mg} / \mathrm{kg} / \mathrm{min}$ to $1.14 \pm 0.20$; 'nonresponders': $1.40 \pm 0.35$ to $1.20 \pm 0.30$; NS) did not evolve differently in the responder and nonresponder groups. Lipid infusion modified postabsorptive glycemia in NIDD patients. The response was heterogeneous, and needed lipolysis of the infused triglycerides. This did not occur the same way in all subjects, as previously shown in normal subjects [Peterson (1990) Proc Natl Acad Sci USA 87, 909]. In contrast, an elevation of lipid oxidation did not seem necessary. The normality of total lipid oxidation in NIDD patients, therefore [De Fronzo (1988) Diabetes 37,667 ], did not exclude a role of lipid-glucid interactions during hyperglycemia. Interaction at the oxidative level did not seem sufficient to elevate glycemia, indicating an additional effect of lipid infusion on endogenous glucose production or nonoxidative glucose disposal.

\section{Effects of glucagon on fructose-induced alterations of glucose metabolism in man. N Paquot ${ }^{1}, \mathrm{P}$ Schneiter ${ }^{2}$, E Jéquier ${ }^{2}$, L Tappy ${ }^{2}$ ( 1 Division of Diabetes, CHU-Sart- Tilman, 4000 Liège, Belgium; 2 Institute of Physiology, University School of Medicine, Bugnon 7, 1005 Lausanne, Switzerland)}

Gluconeogenic substrates increase gluconeogenesis but fail to enhance the overall endogenous glucose production (EGP). The mechanisms responsible for this autoregulation remain unknown. In order to assess the effects of hyperglucagonaemia on autoregulation of EGP, eight healthy sub- 